that time the claims for evidence for the genetic model must be considered as no more than an indication of the biases of the scientists (and philosophers) who put forth the claims.

These arguments apply, of course, to the question of sex roles which Ruse discusses in his penultimate chapter. According to Ruse, one is not a sexist if one values positively the role of the female human being. This extraordinarily naive position ignores the broad cultural and institutional forms of power in society which perpetuate male domination. Sociobiologists place a heavy emphasis on sexual selection and on sex role division of labour and its evolutionary basis. These points are essential to the theory. The sexism of sociobiology does not lie in its conclusions, as Ruse would have us believe the critics have charged. Rather, the conclusions are a natural consequence of the fundamental assumptions which underly the whole sociobiological enterprise. For example, basic to E.O. Wilson's explanation of sex roles as they exist today is a reconstruction of human prehistory which describes the male as a hunter from early evolutionary times. He accepts the arguments of popularizers of palaeontology such as Ardrey and ignores the bulk of evidence which suggests that, for most of hominid evolution, we were vegetarians and fruit eaters. There are numerous similar examples in sociobiology where a social bias leads to the choice of explanation or of data which, in turn, are determinants of the conclusions of the theory. One has only to read Kropotkin's Mutal Aid to see that a different social perspective leads to a choice of different examples and thus to a totally different view of human nature.

This is a strange, contradictory and provocative book. While admitting that "one should [not] try to pretend that science can proceed divorced from ideology', Michael Ruse ignores the pervasive ideological influences on those attempts to use the biological sciences to explain human social behaviour.

Jon Beckwith is in the Department of Microbiology and Molecular Genetics at Harvard Medical School.

\title{
Putting the environment back in genetics
}

\section{Lindon J. Eaves}

Cultural Transmission and Evolution: $A$ Quantitative Approach. By L.L. CavalliSforza and M.W. Feldman. Pp.388. Hbk ISBN 0-691-08280-4; pbk ISBN 0-69108283-9. (Princeton University Press: 1981.) Hbk $\$ 25, £ 17.70$; pbk $\$ 10.50, £ 7.50$.

IN MANY applications of quantitative methods to human genetics it has been assumed, by default, that transmission is biological in spite of repeated criticism that environmental factors may simulate Mendelian inheritance. Historians and philosophers of science may wonder why the genetic theory of human differences has proved so powerful. Some of the credit must be given to the fact that the consequences of genetic inheritance found a precise mathematical form very early, in the work of such pioneers as Fisher and Wright. By contrast, environmental transmission models were inexplicit and devised to explain rather than predict.

The past ten years have seen a number of critical developments which have gone far to redress the imbalance of theory. Sociobiology arose because existing evolutionary theory seemed to run counter to those very human characteristics that evolution had established. Much of this work has been conservative, retaining at its centre the genetic basis of information transfer and attempting to show how such characteristics of higher organisms as sex, altruism, parental care, social organization and mate selection could evolve in a Darwinian universe.
Cavalli-Sforza and Feldman begin from a radically different position. They take the human capacity for learning and social interaction for granted and examine the many ways in which the dynamics and equilibria of human populations are modified when the rules of transmission are freed from their Mendelian straitjacket. Their work is a pleasing balance of theory and colourful examples.

They examine a number of ways in which cultural transmission may differ from genetic inheritance. In the simplest case, transmission may involve a single dimorphic cultural state passed from parent to child without the constraints which accompany Mendelian segregation. Such "vertical transmission" may often be confused with Mendelian inheritance but can generate a far wider range of dynamics and equilibria. The authors show, for example, the conditions under which cultural states may oscillate between generations if children react against the values of their parents. The theory is developed mathematically using assumptions and methods largely familiar to population geneticists. Cultural parallels of mutation, migration, selection and drift are examined, together with some models of assortative mating. Later chapters consider multi-state and continuous traits.

Cavalli-Sforza and Feldman recognize that transmission is not confined to the nuclear family. Information may be passed from teachers in the parental generation ("oblique" transmission) or in the same generation ("horizontal" transmission). In such cases only the type of social organization and communication network restrict the number of people influenced by a new idea, so the rates of evolutionary change increase dramatically. One possible consequence is the rapid spread of an innovation which has an adverse effect on fitness.

The authors are the first to admit that there is an element of unreality in treating cultural transmission independently of genetic inheritance, and there are other workers who have considered the joint effects of genetic factors and social interactions on the resemblance between relatives. Perhaps the only weakness of the present book is a failure to integrate the theory with other contemporary work in human quantitative genetics, much of which is just as concerned with the environment.

Part of the richness of Cultural Transmission and Evolution stems from the inclusion of illustrative examples, ranging from national differences in pronunciation, through the familial transmission of Buddhism, to the distribution of surnames. Data are used by Cavalli-Sforza and Feldman very much as they were used a century ago by Galton and Pearson, to illustrate rather than prove. There is little attempt to test and exclude alternative genetic hypotheses.

The long-term value of the book is likely to stem not from the data, however, but from the authors' ability to crystallize the elements of cultural transmission in a mathematical model which starts with a few simple assumptions. It offers, to biologists and behavioural scientists alike, an attractive demonstration of the heuristic value of model-building as a way of drawing together the more haphazard intuitions of the past and may make clinical and behavioural geneticists more sensitive to the ambiguities of their data.

Lindon J. Eaves is Distinguished Professor of Human Genetics at the Medical College of Virginia, Richmond.

\section{Supergiant behaviour}

\section{J.B. Hutchings}

The Brightest Stars. Geophysics and Astrophysics Monographs, 19. By Cornelis De Jager. Pp.456. Hbk ISBN 90-277-1109-7; pbk ISBN 90-277-1110-0. (Reidel; 1981.) Hbk Df1.140, \$73.50; pbk Dfl.60, \$31.50.

IN attempting to cover our current knowledge of the most luminous stars in nine self-contained chapters, Dr De Jager has set himself a formidable task. The recent pace of research has expanded the frontiers of this subject to an extent that no one volume can fully encompass. What the 